\title{
Mit dem IKRK und MSF in Kriegsgebieten unterwegs
}

\section{Tanja Kühnle}

Dipl.-Biol., Managing Editor

Die letzte Prüfung ist geschafft, das Medizinstudium beendet. Und wie geht es jetzt weiter? In unserer Serie «Du findest deinen Weg!» stellen wir Ihnen in unregelmässigen Abständen aussergewöhnliche Berufsperspektiven für Mediziner vor. In dieser Ausgabe berichtet Dr. Fränzi Auf der Maur von ihren Auslandseinsätzen mit dem Internationalen Komitee vom Roten Kreuz (IKRK) und mit Médecins Sans Frontières (MSF).

Frau Auf der Maur, wollten Sie schon immer für eine Hilfsorganisation arbeiten?

Schon als Kind habe ich Leute bewundert, die sich in Katastrophen- und Krisengebieten engagieren. Dies war ein Mitgrund dafür, dass ich Medizin studiert und mich für Anästhesie als ersten Facharzt entschieden habe.

\section{Wie kam es dazu, dass Sie sich für das IKRK und MSF entschieden haben?}

Als Schweizer Medizinerin ist es naheliegend, kriegschirurgische Einsätze mit dem IKRK und MSF zu machen - beides Organisationen, die in der Schweiz sehr bekannt sind und einen guten Ruf sowie eine lange Tradition haben.

\section{Brauchen Sie eine spezielle Zusatzausbildung für einen solchen Auslandseinsatz?}

Es gibt meines Wissens keinen Kurs für Anästhesie in Kriegsgebieten. Das ist Learning by Doing. Hilfreich für die Arbeit in diesen Ländern, die sich ja nicht nur auf den Operationssaal bezieht, ist sicherlich ein tropenmedizinischer Kurs, den ich jedoch noch nicht gemacht habe.

\section{Wie lange waren Sie jeweils im Einsatz?}

Bisher zwei bis drei Monate. Der nächste Einsatz wird jedoch sechs Monate dauern, da ich ein Angebot als Senior Anaesthetist im Südsudan bekommen habe.

Und in welchen Ländern waren Sie bislang unterwegs? Im Südsudan, im Jemen, in Somalia, in Zentralafrika und im Kongo.

Wie waren Sie dort jeweils untergebracht?

Das ist sehr verschieden und abhängig vom Einsatzgebiet und von der Sicherheitslage vor Ort. Im Feld im Südsudan haben wir wie die Einheimischen in Lehmhüt-

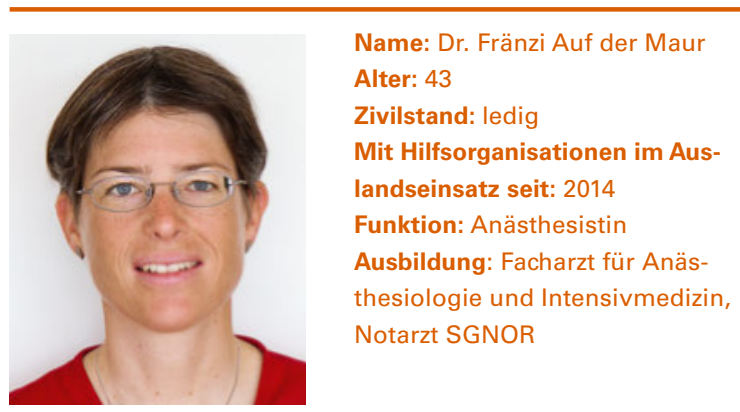

ten geschlafen, dies in einem Einmannzelt aus Moskitonetz. In sehr gefährlichen Gegenden wie Somalia oder dem Jemen schläft man in streng bewachten Häusern, teils mit Sandsäcken vor den Fenstern als Schutz vor Explosionswellen. Bei meinem Einsatz in Somalia war ich aus Sicherheitsgründen in Nairobi stationiert und bin jede Woche ins Land geflogen.

\section{Wie muss man sich einen Operationssaal in einem Kriegsgebiet vorstellen?}

Das ist ganz unterschiedlich. In Städten und grösseren Orten arbeiten wir in bestehenden Spitälern mit einem richtigen Operationssaal, einem verstellbaren Tisch, guten Lampen und fast allem, was man braucht, um gute Medizin zu machen. Im Feld im Südsudan ist es jedoch ein kleiner, dunkler Raum, der nicht dicht verschliessbar ist. Da musste ich jeden Morgen zuerst die Kröten und Skorpione evakuieren, die es sich während der Nacht hinter der Anästhesietasche bequem gemacht haben.

\section{Wird man vom IKRK und von MSF auf die Einsätze vorbereitet?}

Meistens bekommt man im Voraus Informationen über das Land, den Kontext und die Lebensbedingun- 


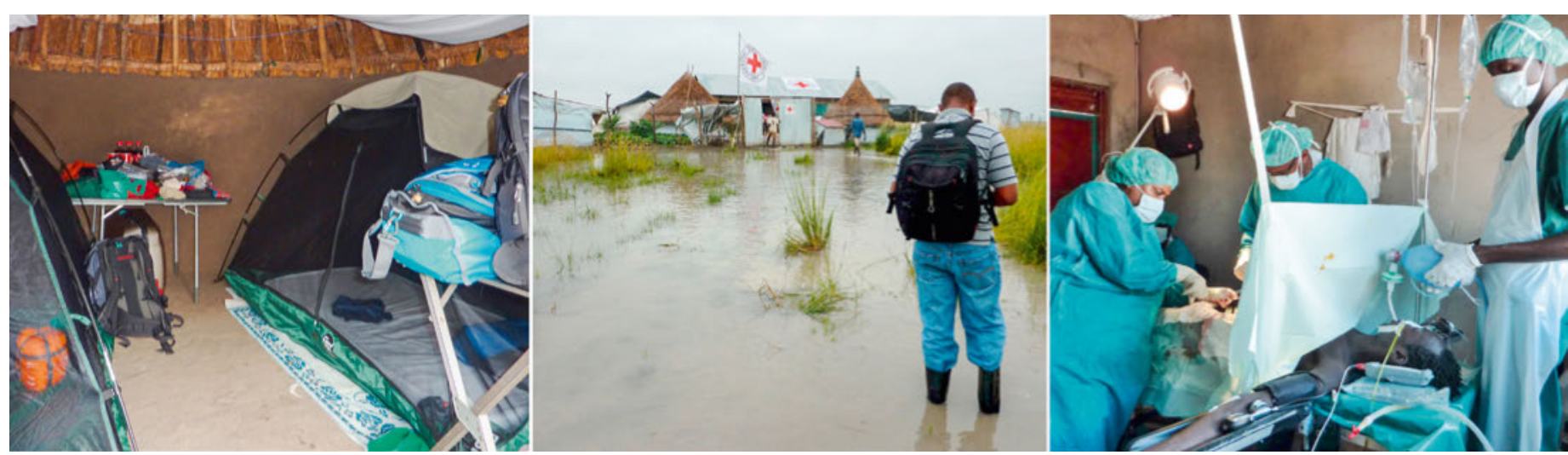

Im Südsudan war Fränzi Auf der Maur zusammen mit einer Kollegin in einem Zelt in einer Lehmhütte untergebracht. Während der Regenzeit stand das Gelände rund um das Spital manchmal knöcheltief unter Wasser. Das Foto rechts gewährt einen Blick in den sehr einfachen Operationssaal.

gen vor Ort. Vieles muss man dann jedoch auf sich zukommen lassen. Am Tag vor dem Abflug gibt es ein Briefing im Hauptsitz in Genf, danach noch einmal detaillierter nach der Ankunft im Einsatzland. Auf denjenigen Einsätzen, wo man selber autofahren darf, muss man jedes Mal wieder erneut einen Fahrtest mit den Toyota Land Cruisern machen. Beim ersten Einsatz war ich zu Beginn überfordert mit der Tatsache, dass ich nicht wusste, wie ich ohne Beatmungsmaschine, Anästhesiegas und Spritzenpumpe eine Narkose aufrechterhalten kann. Nach einem Tag mittelmässiger Einführung durch einen Kollegen vor Ort war ich dann ganz auf mich alleine gestellt.

\section{Bereiten Sie sich auch selbst auf die Einsätze vor?}

Ja. Ich kaufe mir immer eine Karte und suche im Internet nach Informationen und Bildern über die Gegend, wo ich stationiert sein werde.

\section{Wie war es mit der Verständigung in diesen Ländern?} Ich spreche Deutsch, Englisch und Französisch und habe immer ein Wörterbuch in der jeweiligen Landessprache dabei. Ausserdem lege ich mir stets ein kleines Repertoire an Wörtern und einfachen Sätzen zu, damit ich den Patienten selbst die wichtigsten Fragen stellen kann. Das wird extrem geschätzt, sowohl von den Patienten und deren Angehörigen wie auch von den Mitarbeitern, die einiges zu lachen hatten, als ich meine ersten Gehversuche auf Suaheli oder Arabisch gemacht habe.

\section{Können Sie denn auch einen Übersetzer hinzuziehen?} Das ist sehr abhängig vom Einsatz. Falls die lokalen Mitarbeiter sehr gut Englisch oder Französisch sprechen, ist dies in der Regel nicht nötig. Im Südsudan, im Jemen und in Somalia standen uns jedoch rund um die Uhr Übersetzer zur Verfügung, da sonst weder die Kommunikation mit dem Personal noch jene mit den Patienten gewährleistet gewesen wäre.

\section{Nimmt man auf solche Einsätze auch eigenes Equipment mit?}

Nein, aus Standard-Gründen sollten alle Mitarbeiter mit demselben Material arbeiten. Die lokalen Mitarbeiter der Sterilisation wissen genau, welche Instrumente in welches Set gehören, so dass es zu Verwirrungen führen würde, wenn jeder Chirurg zusätzlich seine eigenen dabei hätte.

\section{Und wie sieht es mit der Kleidung aus?}

Es gibt vor Ort OP-Kleidung und Schuhe für uns, nur manchmal nicht in der richtigen Grösse. Wir können aber auch unsere eigenen Kleider mitnehmen. Und die spezielle Kleidung, die man als Frau in arabischen Ländern benötigt, wird gestellt. Schon vor der Anreise wird man mit mindestens einem Set ausgestattet. Und im Laufe des Einsatzes kommt man dann zu immer mehr. Im Jemen zum Beispiel gab es in unserem Wohnhaus eine Kiste mit verschiedenen schwarzen Kleidern und Kopftüchern, wo wir Frauen uns bedienen konnten.

\section{War es für Sie unangenehm, mitten im Sommer bodenlange Kleider und ein Kopftuch zu tragen?}

Ja, denn es war häufig sehr heiss. Vor allem in Somalia habe ich in dieser Kleidung sehr gelitten.

\section{Was sind die häufigsten Verletzungen, die Sie behandelt haben?}

Das ist je nach Einsatz unterschiedlich. Im Jemen gab es beispielsweise viele Verletzungen durch Bomben und Granaten. In Zentralafrika und im Kongo kamen neben den Schussverletzungen, die es überall gibt, viele Machetenverletzungen dazu. Da in der Kriegschirurgie aufgrund des Infektionsrisikos keine interne Osteosynthese durchgeführt wird, werden Femurfrakturen durch Extension und die anderen Frakturen meist mit einem Fixateur extern versorgt. Wundinfektionen und Osteomyelitiden sind leider häufig. 


\section{Was war Ihre bislang grösste Herausforderung auf einem Auslandseinsatz?}

Im Jemen haben wir extrem viel gearbeitet, manchmal nächtelang. Während 60 Stunden habe ich einmal insgesamt nur sechs Stunden geschlafen. Dies führt dann natürlich aufgrund der Übermüdung auch zu Fehleranfälligkeit.

\section{Und gab es auch eine besondere medizinische Herausforderung?}

Medizinisch gesehen gab es sehr viele Herausforderungen. Vor allem deshalb, weil man viel improvisieren muss. In Zentralafrika habe ich beispielsweise die erste Peritonealdialyse im Land überhaupt gemacht. Wir hatten einen jungen Patienten, der nach einer blanden Laparotomie nach stumpfem Abdominaltrauma über mehrere Tage anurisch blieb, ohne dass wir eine Ursache erkennen konnten. Aus Verzweiflung habe ich eine E-Mail an mehrere Kollegen in der Schweiz geschrieben, ob sie mir einen Nephrologen empfehlen könnten, der mir weiterhelfen würde. Innerhalb von 24 Stunden haben sich sieben Nephrologen aus der ganzen Schweiz gemeldet, von denen ich nur einen kannte. Diese Solidarität hat mich ungemein gerührt. Unter Anweisungen des Chefs der Dialyse am Inselspital Bern haben wir dann mit den vorhandenen Infusionsflüssigkeiten eine Lösung konstruiert, die wir mehrmals täglich über zwei in den Bauch eingelegte Blasenkatheter infundiert und wieder abgelassen haben. Die Diurese kam langsam zurück, und das ganze Team, das mit viel Elan an diesem Projekt mitgeholfen hat, war stolz auf unseren Erfolg. Leider ist der Patient dann vor meinen Augen an einer oberen Gastrointestinalblutung verstorben.

\section{Wie kommen Sie persönlich mit diesen vielen, furchtbaren Eindrücken zurecht?}

Für mich ist es immer besonders schlimm, wenn Kinder unter dem Krieg leiden. Im Kongo haben wir ein vierjähriges Mädchen bekommen, dem mit einer Machete der Schädel gespalten wurde, als das Dorf, in dem es und seine Familie lebten, überfallen wurde. Wir haben die Wunden versorgt, doch das Kind war schwer traumatisiert, so dass sich niemand dem Bett nähern konnte, ohne dass es zu schreien begonnen hat. Mit viel Zuwendung - und auch mal ein paar Süssigkeiten hat es dann zunehmend Vertrauen geschöpft, wurde kommunikativ und hat jeden angestrahlt, so dass es zum Liebling des Spitals geworden ist.

Ein anderes Beispiel bezieht sich auf die Tatsache, dass wir rein aufgrund der limitierten Ausrüstung Patienten sterben lassen müssen. Es war im Jemen, wo wir eines Abends erneut viele Schwerverletzte bekamen, darunter ein junger Mann mit thorakaler und abdominaler Schussverletzung. Seine Oxigenation war miserabel, und dies mit 100\% Sauerstoff am Respirator. Auf dem Notfall haben weitere fünf Patienten mit abdominalen Schussverletzungen gewartet. Ich hätte ihn unmittelbar nach der Operation extubieren müssen, da wir keine Beatmungsmaschinen auf der Intensivstation hatten, was aber seinen sicheren Tod bedeutet hätte. Da habe ich in einer Kiste ein Peep-Ventil gefunden, es an den Beatmungsbeutel geschraubt und den intubierten, spontan atmenden Patienten damit auf die Intensivstation gegeben. Er hat sich damit sehr gut erholt, so dass ich ihn am nächsten Morgen extubieren konnte. Unkonventionell, doch es hat funktioniert.

Solche Erfolge machen Freude und das Improvisieren Spass.

\section{Erinnern Sie sich auch an besonders schöne Begegnungen mit Menschen vor Ort?}

Ja, ich habe viele tolle Bekanntschaften gemacht. Zum Beispiel habe ich im Jemen ein zwölfähriges Mädchen kennengelernt, das auf dem Schulweg angeschossen wurde. Es war zum Glück nicht lebensgefährlich verletzt, musste aber wegen Wundspülungen immer wie-
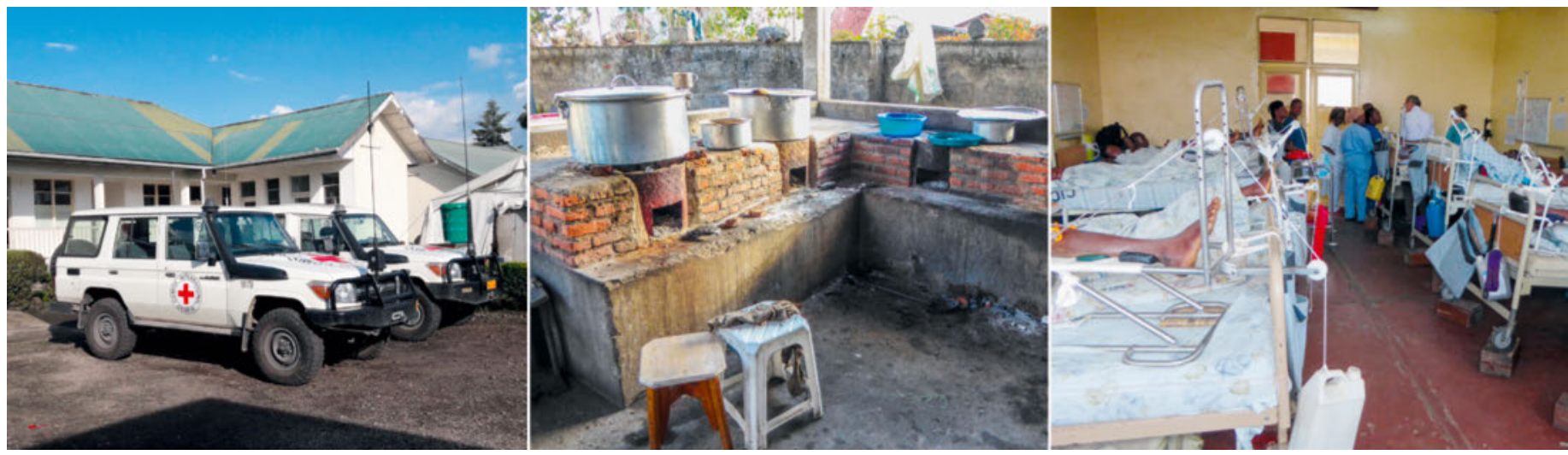

Das Spital in Goma (Kongo). Auf dem Foto in der Mitte ist die Spitalküche zu sehen. Das rechte Bild zeigt ein Zimmer, wo die meisten Patienten mit einer Traktion für zwei bis drei Monate ans Bett gefesselt sind. 


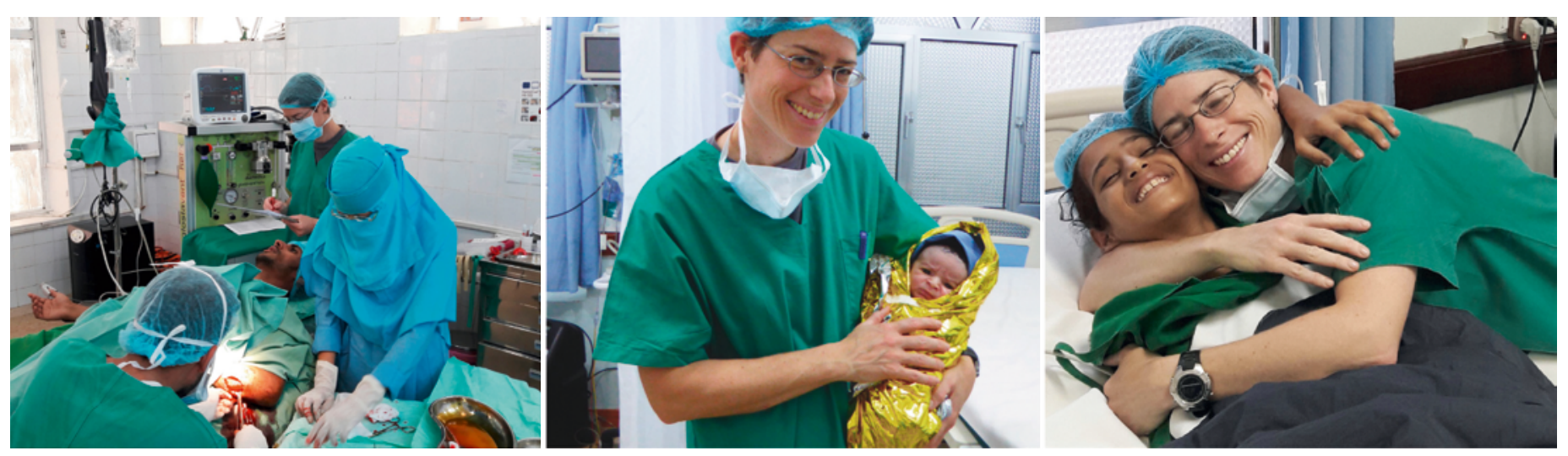

Der Operationssaal im Spital im Jemen (links). Neben den vielen traurigen gab es auch schöne Momente - zum Beispiel, wenn ein Kind per Kaiserschnitt das Licht der Welt erblickt hat oder wenn ein verletztes Kind (12-jähriges Mädchen mit Schussverletzung) nach der OP wieder lachen konnte.

der in den OP kommen. Wir haben uns von Anfang an gut verstanden, obwohl wir uns aufgrund der Sprachbarriere nur mit Mimik und Gesten verständigen konnten. Als ich ihr dann gesagt habe, dass ich das nächste Mal nicht mehr hier sein würde, hat sie bitter geweint. Auch die Dankbarkeit der Patienten, Angehörigen und lokalen Mitarbeiter tut gut. Ich bekomme aus mehreren Einsatzländern immer noch regelmässig Nachrichten, was mich sehr freut.

Sind Sie auf einem Einsatz mal selbst in Gefahr geraten? Beide Organisationen sind extrem auf Sicherheit bedacht. Einmal wurden wir aus einem Spital evakuiert, weil über das Gebäude geschossen wurde. Es gab dann eine kurze Feuerpause, damit wir das Haus verlassen konnten. Es kommt immer wieder vor, dass wir aufgrund der Sicherheitslage im Ort oder ums Spital gar nicht arbeiten gehen können. Doch hierfür gibt es eine Sicherheitsabteilung, die uns darüber informiert. Man bekommt den Krieg also schon nah mit, aber ich bin noch nie bedroht worden oder in Gefahr geraten. In einigen Destinationen ist Kidnapping ein Problem, zum Beispiel in Somalia. Deshalb durften wir uns dort nur in einem bewaffneten Konvoi bewegen, und ich wurde auch im Spital immer von bewaffneten Sicherheitsleuten begleitet. Aber auch während dieses Einsatzes hatte ich nie Angst um mein Leben.

\section{Sind Sie schon einmal an einen Einsatzort zurückgekehrt?}

Nein. Ich habe zwar immer gesagt, dass ich gerne zurückgehen würde, da es immer gute Einsätze waren. Aber bislang haben sie mich immer woanders hingeschickt, was es auch spannend macht.

\section{Gab es etwas, das Sie während der Einsätze besonders vermisst haben?}

Mir haben meine Familie, meine Freunde und meine
Hobbys gefehlt. Ich mache selbst viel Musik, gehe gerne ins Konzert oder ins Theater und verbringe jeden freien Tag in den Bergen. Das geht auf so einem Einsatz natürlich nicht. Dazu kommt, dass die Expats aus aller Herren Länder kommen und aufgrund der unterschiedlichen Interessen sich Diskussionen manchmal schnell erschöpfen. Man muss sich also sehr gut selbst unterhalten können. Ich habe mir auch ein paar Übungen zugelegt, die ich auf beschränktem Platz und mit einem Thera-Band machen kann, da man sich an vielen Orten nicht frei bewegen kann.

\section{Gibt es noch etwas, das Sie jungen Kolleginnen und Kollegen raten würden, die auch auf einen solchen Auslandseinsatz gehen möchten?}

Man muss sich bewusst sein, dass man auf einem Einsatz auf vieles verzichten muss, was einem zu Hause wichtig ist. Je nach Land lebt man sehr einfach und muss sich als Frau den dortigen Kleidervorschriften anpassen. Man muss flexibel und tolerant sein und gerne improvisieren. Auf der anderen Seite sind solche Einsätze sehr befriedigend. Man führt zusammen mit dem Chirurgen und den Pflegenden, je nach Einsatzort unterstützt durch einen Projekt Manager, ein ganzes Spital mit vielen Mitarbeitern und kann sehr viel bewirken und entscheiden. Dazu lernt man viel über fremde Kulturen und Religionen und knüpft bleibende Kontakte zu Menschen in unterschiedlichsten Ländern, seien es nun Expats oder lokale Mitarbeiter.

\section{Bildnachweis}

Fotos: Fränzi Auf der Maur 